Check for updates

Cite this: Nat. Prod. Rep., 2019, 36 1044

Received 4th December 2018

DOI: $10.1039 / c 8 n p 00096 d$

rsc.li/npr

\section{The protosteryl and dammarenyl cation dichotomy in polycyclic triterpene biosynthesis revisited: has this 'rule' finally been broken? $\uparrow$}

\author{
Michael J. Stephenson, (D) Robert A. Field (D) and Anne Osbourn (D)* \\ Covering: 1948 up to the end of 2018
}

The triterpene alcohols represent an important and diverse class of natural products. This diversity is believed to originate from the differential enzymatically controlled cyclisation of 2,3-oxidosqualene. It is now a wellestablished presumption that all naturally occurring tetra- and penta-cyclic triterpene alcohols can be rationalised by the resolution of one of two intermediary tetracyclic cations, termed the protosteryl and dammarenyl cations. Here, a discussion of typical key triterpene structures and their proposed derivation from either of these progenitors is followed by comparison with a recently reported novel pentacyclic triterpene orysatinol which appears to correspond to an unprecedented divergence from this dichotomous protosteryl/dammarenyl view of triterpene biogenesis. Not only does this discovery widen the potential scope of triterpene scaffolds that could exist in nature, it could call into question the reliability of stereochemical assignments of some existing triterpene structures that are supported by only limited spectroscopic evidence. The discovery of orysatinol provides direct experimental evidence to support considering more flexibility in the stereochemical interpretation of the biogenic isoprene rule.

\section{Introduction}

The triterpenes represent an important and diverse class of natural products, and one which has captured the interest of organic chemists for the best part of a century. Hundreds of unique basal triterpene scaffolds have been isolated from nature. ${ }^{1}$ Despite this observed diversity, all triterpene alcohols are believed to originate from the same linear precursor, known trivially as 2,3-oxidosqualene (1). Indeed, since the inception of the biogenic isoprene rule in the $1950 \mathrm{~s},{ }^{2,3}$ this skeletal diversity has been rationalised through mechanistic reasoning to arise from the differential enzymatically controlled cyclisation of this common substrate or from squalene itself. With advances in genomic and transcriptomic sequencing, and the advent of convenient heterologous expression platforms, an increasing number of the enzymes that mediate this process (oxidosqualene cyclases, OSCs) have now been cloned and functionally characterised, strengthening further this already wellestablished biogenic presumption. ${ }^{4}$

One of the richest sources of triterpene diversity is the plant kingdom, where this family of natural products serve as precursors to many important and structurally diverse

John Innes Centre, Norwich Research Park, Norwich, NR47UH, UK. E-mail: anne. osbourn@jic.ac.uk

$\dagger$ Electronic supplementary information (ESI) available. See DOI: 10.1039/c8np00096d secondary metabolites. ${ }^{5,6}$ However, triterpenes derived from 2,3oxidosqualene (1) are also essential primary metabolic precursors in seemingly all eukaryotic kingdoms. A terminological distinction is often made (particularly in phytology) between triterpenes implicated in secondary metabolic pathways and those originating from primary metabolism, the latter being commonly referred to as sterols. ${ }^{4}$ This distinction also has chemical significance, since sterols fundamentally differ in the stereochemical configurations of their ring architectures. This is exemplified by comparison of the two constitutionally identical tetracyclic compounds lanosterol $(4)^{7-9}$ and euphol (5). ${ }^{10}$ These two compounds differ only in the configuration of three (out of seven) stereogenic centres. This is most strikingly evident in the opposite relative configuration of the attachment of the methyl groups at the C/D trans ring junctions (Fig. 1).

The structural comparison between lanosterol (4) and euphol (5) is of historical significance, as it was attempts to rationalise the differing stereochemical outcome cemented in the final scaffolds of this pair of natural products that would eventually result in the 1950s postulation of two distinct and fundamental cyclisation routes..$^{1-3,11,12}$ It was proposed that in the case of lanosterol (4), the series of initial electrophilic additions resulting in the first three ring-forming annulations proceed via a chair-boatchair conformational folding. Conversely, the equivalent steps in the formation of euphol (5) proceeds via the less sterically demanding chair-chair-chair conformation. Subsequent formation of the D rings results in two distinct tetracyclic carbocations, 
now termed the protosteryl (2) and dammarenyl (3) cations respectively (Fig. 1). ${ }^{\mathbf{1} 4}$ All naturally occurring triterpenes alcohols known at that time were subsequently reasoned to originate from one of these two key intermediates.

Over the proceeding decades, many more triterpene and sterol scaffolds presumed to arise from the cyclisation of 2,3-oxidosqualene (1) have been isolated from nature, and consistently their formation has been rationalised on the basis of this dichotomous pair of cyclisation pathways. The precise intricacies of these cyclisation mechanisms, and their enzymatic control, is still an active area of research. ${ }^{13-15}$ However, it is now a wellestablished and perhaps dogmatic presumption that to date all naturally occurring tetra- and penta-cyclic triterpenes derived from 2,3-oxidosqualene (1) can be rationalised by, or predicted to arise from, the eventual resolution of either the protosteryl cation (2) or the dammarenyl cation (3). The differential resolutions of these two cations, are typically proposed to proceed via further

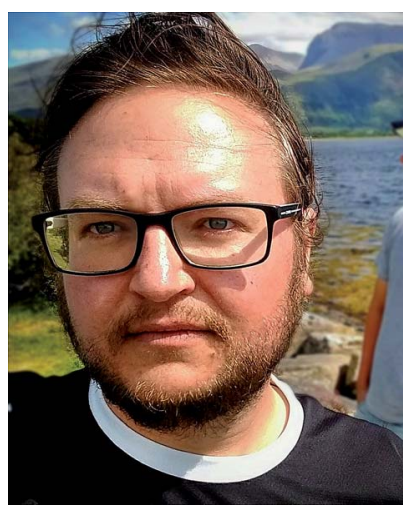

Michael Stephenson received his MPharm degree in 2010 and qualified as a registered pharmacist the following year. Michael then returned to academia to pursue a PhD in medicinal chemistry within Prof. Mark Searcey's group at the University of East Anglia. This was awarded in 2015 for the development of novel methodology for the rapid synthesis of analogues of the potent antitumor-antibiotic duocarmycin. Since then Michael has been a postdoctoral scientist in Prof. Anne Osbourn's group at the John Innes Centre, where his research has focused on the utilisation of transient plant expression for the preparative production of highvalue triterpenes.

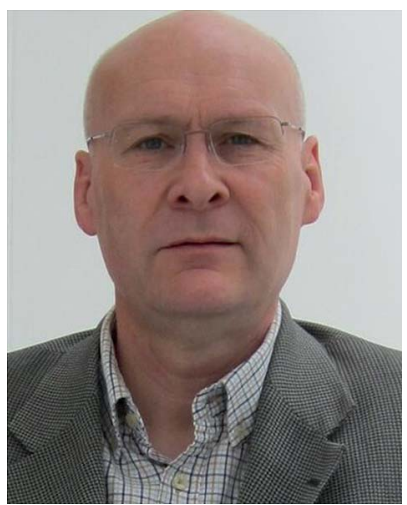

Rob Field is a University of East Anglia graduate (1986) and PhD (1989; $\mathrm{Dr}$ A. H. Haines). Following postdoctoral work in Oxford (1989-91; Prof. J. E. Baldwin), Dundee (1992-94; Prof. M. A. J. Ferguson and Prof. S. W. Homans) and Alberta (1994; Prof. O. Hindsgaul), he was appointed to the faculty at the University of St Andrews in 1994, where he was promoted through the ranks to Professor (1999). He returned to Norwich in 2001, initially at University of East Anglia and latterly at the John Innes Centre. His research programme focuses on plant and microbial carbohydrate chemistry, in particular cell wall biosynthesis, starch metabolism and natural product biosynthesis. 1,2-hydride and alkyl shifts, with or without additional ringforming or -expanding rearrangements. The reactions are typically terminated by vicinal hydrogen elimination to form an alkene, although water capture (resulting in diols) and more complex fragmentations are also observed. ${ }^{\mathbf{1 1 6}}$

This interpretation of triterpene biosynthesis is now so wellestablished that it is generally considered a prerequisite during structural characterisation that novel triterpenes should comply with this stereochemical conformity, ${ }^{3}$ a view which is presented in many authoritative reviews. Indeed, this compliance was exhaustively presented for all structures known in 2004 by Xu et al. ${ }^{1} \mathrm{~A}$ historical perspective on the formulation of the biogenic isoprene rule, and its important impact over the proceeding 50 years was given by two of the original authors, Eschenmoser and Arigoni, in $2005 .^{3}$ Some of the more unusual structures rationalised as post-cyclisation fragmentation products of enzymes exploiting the protosteryl (2) or dammarenyl (3) cations were included in a 2008 review by Domingo et al. ${ }^{\mathbf{1 6}}$ To illustrate these points, we present here a small selection of key structures and their relation to either the protosteryl (2) or dammarenyl (3) cation, before going on to highlight the fundamental significance of a novel OSC product reported in 2018 which appears to be the first example of a triterpene which clearly breaks this dichotomy. This discovery raises the possibility that nature utilises a wider range of chemical space in the biosynthesis of polycyclic triterpenes than is currently presumed. It also provides direct experimental evidence to support consideration of more flexibility in the stereochemical interpretation of the biogenic isoprene rule.

\section{Protosteryl-derived compounds}

The protosteryl cation (2) is most closely associated with primary metabolism, ${ }^{4}$ and it is most commonly the progenitor of tetracyclic sterols. ${ }^{1}$ However, the tetracyclic sterols are also the precursors to some secondary metabolites in plants, such as

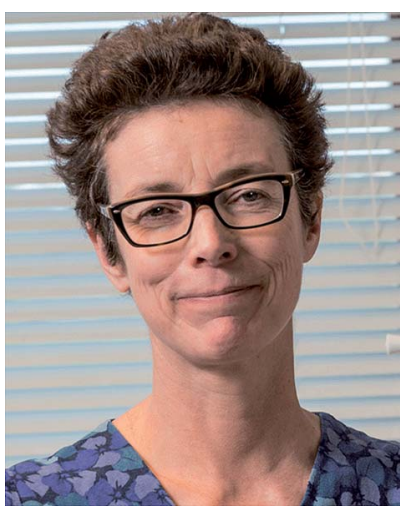

Anne Osbourn is a project leader at the John Innes Centre, an honorary professor at the University of East Anglia, and Director of the Norwich Research Park Industrial Biotechnology Alliance. Her research focuses on plant-derived natural products function, synthesis, and mechanisms of metabolic diversification. An important advance from the Osbourn laboratory has been the discovery that genes for specialized metabolic pathways are organized in 'operon-like' clusters in plant genomes, a finding that has opened up new opportunities for elucidation of new pathways and chemistries through genome mining. Anne also developed and leads SAW, a cross-curricular science education programme (http:// www.sawtrust.org). 


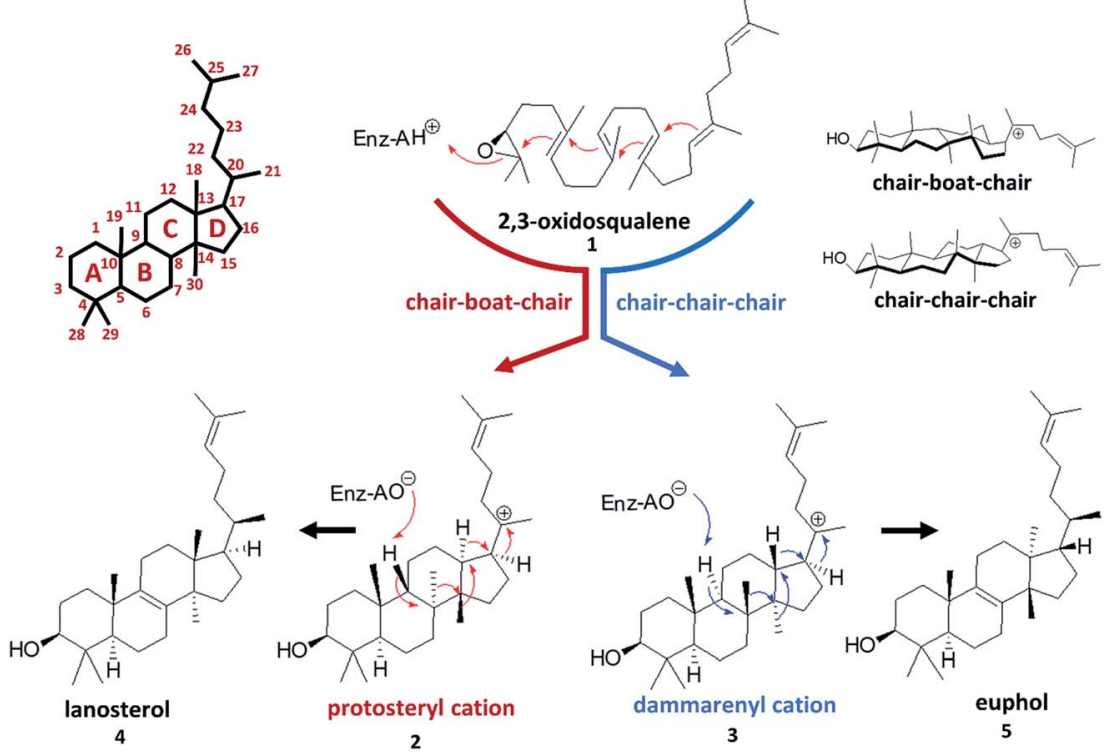

Fig. 1 Structures of 2,3-oxidosqualene, lanosterol, euphol, the protosteryl cation, and the dammarenyl cation. Representation of the established dichotomic initial 2,3-oxidosqualene cyclisation pathways.

the steroidal alkaloids, ${ }^{17}$ and pentacyclic compounds derived from the protosteryl cation (2) are known., ${ }^{1,18-21}$

Lanosterol (4) (whose derivation from the protosteryl cation (2) has already been discussed in the Introduction and Fig. 1) is one of the best studied sterols, being the first committed step in the biosynthesis of cholesterol ${ }^{22}$ and the steroid hormones in mammals. ${ }^{23}$ It is one of the few structures for which the proposed cyclisation mechanism has been well studied, and that is extensively supported by direct experimental and computational evidence spanning many decades. ${ }^{24,25}$ This includes confirming the existence of the protosteryl cation (2) through trapping of its ring structure in lanosterol synthase feeding experiments with substrate mimics. ${ }^{26}$ This work also supported a $\beta$-configuration of the protosteryl cation (2) sidechain contrary to the originally proposed structure which predicted this to be in the $\alpha$-configuration. ${ }^{2}$

Lanosterol (4) is also produced in some higher plants, ${ }^{27}$ but the main protosteryl-derived tetracycles in this kingdom are cycloartenol $(7)^{28,29}$ and parkeol $(8){ }^{30}$ Both compounds can be derived from the same $\mathrm{C} 9$ tetracyclic cation (6), which results after a series of 1,2-hydride and alkyl shifts from the protosteryl cation (2) (Fig. 2a). In the case of parkeol (8), a C11 hydrogen elimination gives the C9-C11 alkene. Whereas, for cycloartenol (7), a hydrogen elimination at the neighbouring methyl group can be invoked to afford the characteristic cyclopropane ring (Fig. 2a).

Pentacycles are also known to originate from the protosteryl cation (2), with isoarborinol (11) $)^{31}$ being one such example. Dring expansion of the protosteryl cation (2) through C13-C17 bond migration gives a 6,6,6,6 tetracyclic cation (9). Subsequent ring closure affords the $6,6,6,6,5$ pentacyclic C22 cation (10). A series of 1,2-hydride and alkyl shifts, terminating in C11 hydrogen elimination gives the alkene (Fig. 2a).

\section{Dammarenyl-derived compounds}

The dammarenyl cation (3) is usually believed to be exclusively harnessed in secondary metabolism. ${ }^{4}$ It is most closely associated with pentacyclic compounds, ${ }^{1}$ but tetracycles ${ }^{1}$ are also known, for example, the previously described euphol (5) (Fig. 1). It is noteworthy that two epimeric forms of the dammarenyl cation (3) differing in the configuration of side chain attachment are postulated, although the term dammarenyl cation (3) is often used trivially without specifying which epimer is being referred to. The $\alpha$-epimer (20) can be invoked to easily rationalise the formation of the C20 epimer of euphol (5), known as tirucallol (21) ${ }^{32,33}$ (Fig. 2b).

Lupeol (14) ${ }^{34}$ and $\beta$-amyrin $(\mathbf{1 6})^{35,36}$ are perhaps the archetypal pentacyclic triterpenes derived from the dammarenyl cation (3). They share a common origin following D-ring expansion via $\mathrm{C} 16-\mathrm{C} 17$ bond migration. Annulation by nucleophilic attack of the side-chain alkene forms the E-ring giving the 6,6,6,6,5 C20 cation (13). In the case of lupeol (14), vicinal hydrogen elimination from one of the geminal methyl groups affords the characteristic terminal alkene of lupeol (14). For $\beta$-amyrin (16), E-ring expansion, followed by 1,2-hydride shifts terminating in C12 hydrogen elimination gives the 6,6,6,6,6 oleanane scaffold (so named due to the most common oxidised form of $\beta$-amyrin (16), the C28 acid, oleanolic acid $\left.{ }^{35}\right)$ (Fig. 2b).

C13-C17 bond migration analogous to that seen in the formation of the isoarborinol-type compounds from the protosteryl cation (2) is also seen with the dammarenyl cation (3). Motiol (19) $)^{37,38}$ is an example of a 6,6,6,6,5 triterpene derived from this route. Again E-ring formation is followed by a series of 1,2-hydride and alkyl shifts, terminated in C7 hydrogen elimination to give the alkene (Fig. $2 \mathrm{~b}$ ). 

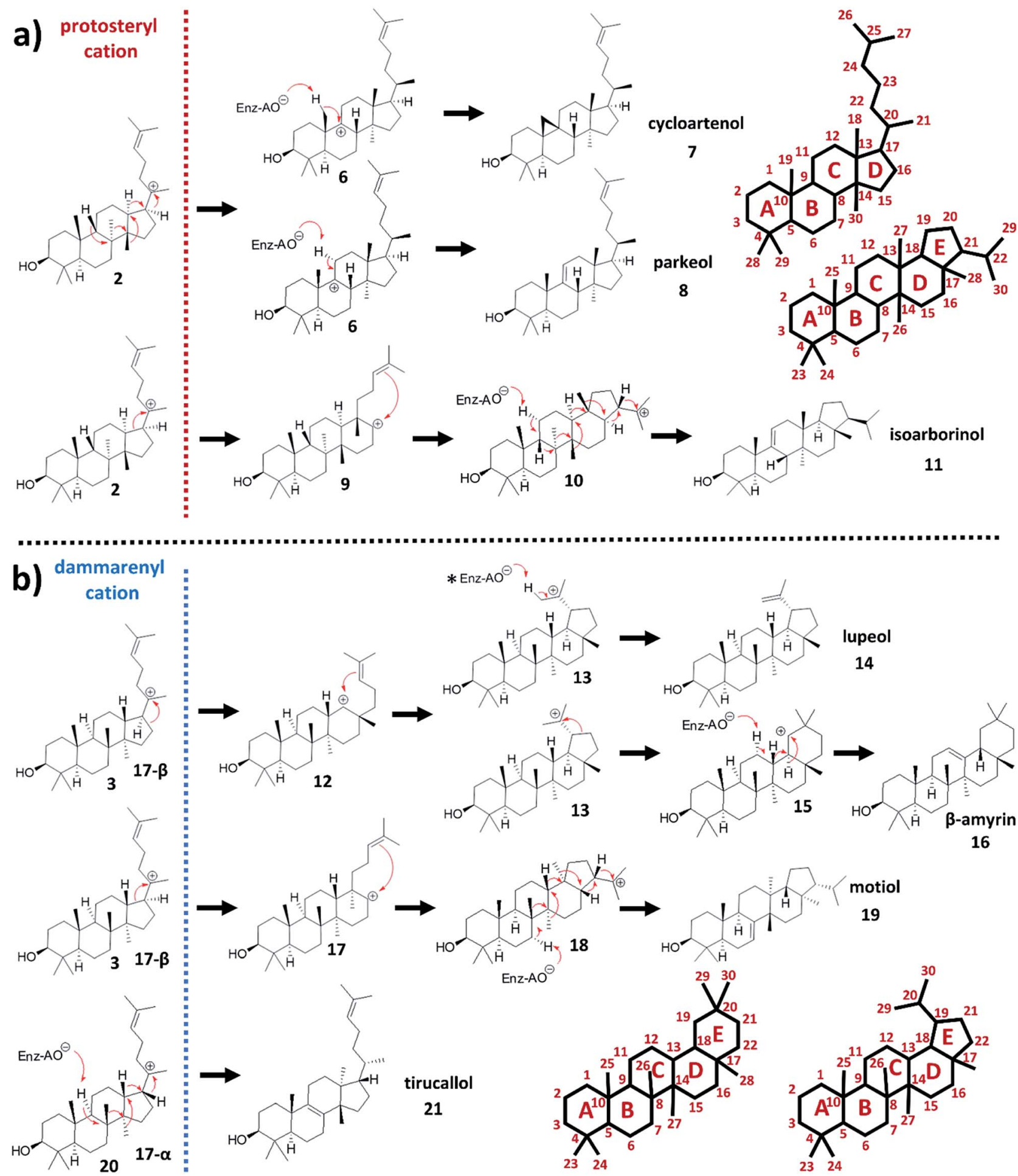

Fig. 2 (a) Key protosteryl-derived compounds. (b) Key dammarenyl-derived compounds. *Deprotonation steps may be spontaneous in same cases.

\section{Many predictable variants of these structures have been isolated from nature}

The majority of other known tetra- and penta-cyclic triterpenes are variations of these basic ring architectures, representing different termination points of 1,2-shift sequences. Many of the scaffolds that would be easily predicted by assuming these sequences follow the tertiary carbon backbone of their respective ring systems have already been isolated from nature. ${ }^{1}$ For example, of the ten alkenes predictable from rearrangement of the oleanane-type 6,6,6,6,6 ring scaffold, nine have been isolated from nature: $\delta$-amyrin $(23),{ }^{34,39}$ germanicol (24), ${ }^{40,41}$ 

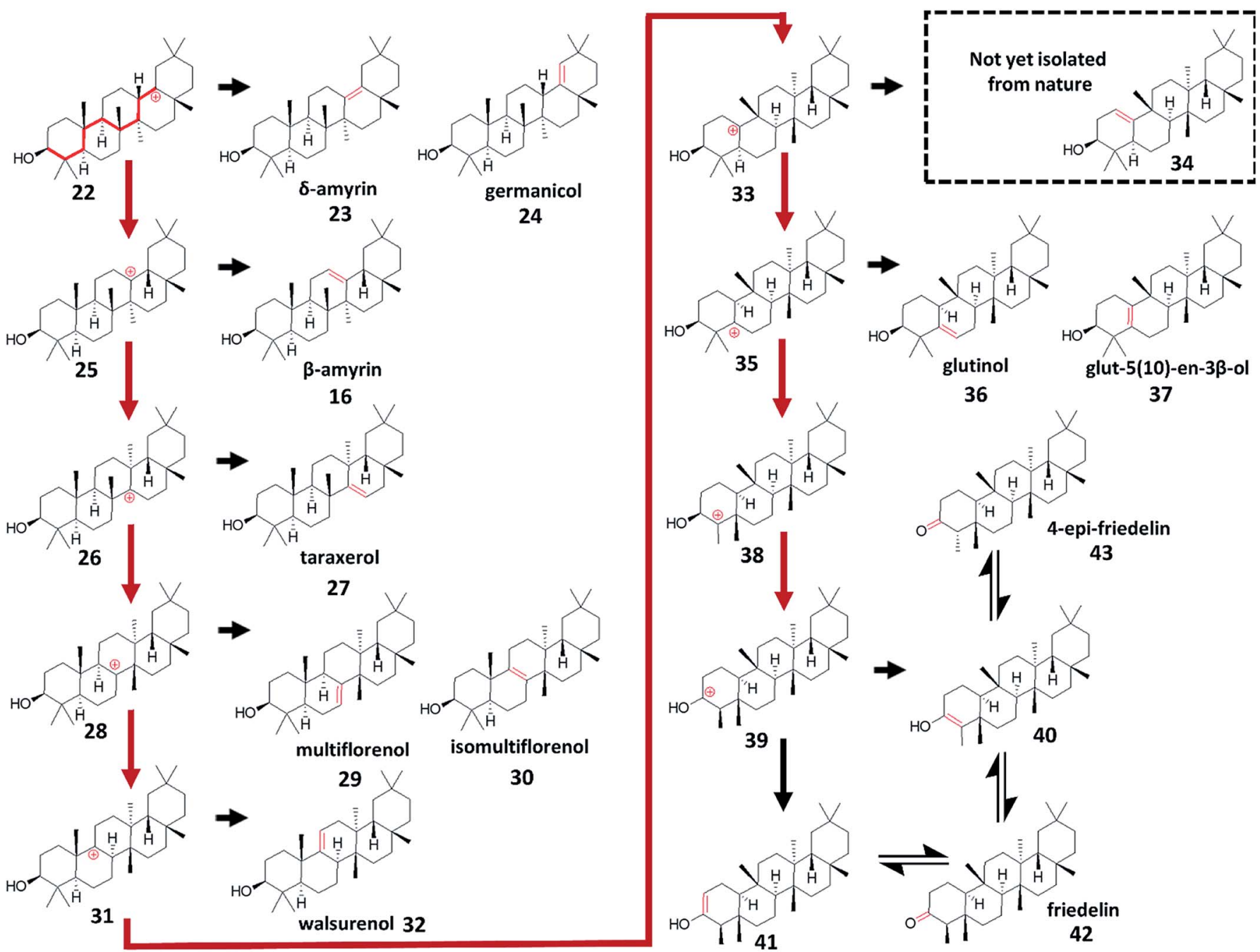

Fig. 3 The ten alkene products which are predictable by assuming the sequence of 1,2-shifts follows the tertiary carbon backbone of the $6,6,6,6,6$ oleanane-type ring system, and the A-ring enols/ketones.

$\beta$-amyrin (16), ${ }^{35,36}$ taraxerol (27), ${ }^{42}$ multiflorenol $(29),{ }^{43}$ isomultiflorenol (30), ${ }^{\mathbf{4 3 , 4 4}}$ walsurenol (32), ${ }^{45}$ glut-5(10)-en-3 $\beta$-ol (37), ${ }^{46}$ and glutinol (36) ${ }^{47,48}$ The two A-ring enols have also been isolated as their respective ketones friedelin (42), ${ }^{49,50}$ and 4-epifriedelin (43) $)^{51}$ (Fig. 3).

\section{Breaking the protosteryl/ dammarenyl dichotomy}

A recent discovery from rice (Oryza sativa L.) $)^{52}$ would appear to represent an explicit divergence from the protosteryl/ dammarenyl dichotomy, and thus may widen the scope of natural triterpenoid diversity that could be hypothesised to exist. The paper focuses primarily on mutagenesis of likely key residues important in the functional conversion of a known rice OSC, ${ }^{53}$ that produces the tetracyclic parkeol $(8)^{30}$ scaffold, to a variant OSC that produces a pentacyclic compound. The pentacyclic compound is a novel natural product which was characterised as the primary product of a divergent OSC discovered from mining the Oryza sativa L. genome. The compound (now named orysatinol (48)) possesses a pentacyclic $6,6,6,6,5 \mathrm{ring}$ scaffold, and is constitutionally identical to the known triterpene motiol (19). ${ }^{37,38}$ However, whereas motiol (19) contains the expected trans $\mathrm{C} / \mathrm{D}$ ring junction rationalisable from the dammarenyl cation (3) (Fig. 2b), orysatinol (48) has an unprecedented cis C/D ring architecture, which has been confirmed by X-ray crystallography (Fig. 4a).

This unprecedented discovery could represent a transformative divergence from the long-established choice between the expected protosteryl (2) and dammarenyl (3) cation cyclisation routes, as production of orysatinol (48) is not easily rationalisable from either of these cations. Conventional reasoning dictates that the methyl groups at the C13 and C14 positions of orysatinol (48) originate from the C14 and C8 positions of the progenitor tetracyclic C20 cation (44) (Fig. 4a). This is the result of a series of suprafacial 1,2-shifts (retaining the stereochemical configuration of the migrating bond) from the pentacyclic $\mathrm{C} 22$ cation (46); a rearrangement that is analogous in sequence to the previously described formation of the 
dammarenyl-derived motiol (19) (Fig. 2b), and protosterylderived isoarborinol (11) (Fig. 2a). As both of these methyl groups are in the $\beta$-configuration, the progenitor tetracyclic C20 cation (44) cannot be either the protosteryl (2) or dammarenyl (3) cation without invoking a stereochemical conversion of the C8 originating methyl group in proceeding steps (Fig. 4a). Therefore, we propose that orysatinol (48) originates from a novel hitherto unpredicted tetracyclic cation, the orysatinyl cation (44), distinct from the protosteryl (2) and dammarenyl (3) cations (Fig. 4a).

Mechanistic reasoning would suggest that the orysatinyl cation (44) should be a C8 epimer of the protosteryl cation (2) (Fig. 4a). This is supported by site-directed mutagenesis work ${ }^{52}$ which report the functional conversion of a committed parkeol synthase to a variant producing orysatinol (48). Further support comes from phylogenetic analysis suggesting that the wild type orysatinol synthase shares a common (parkeol (8) producing) ancestor with the committed parkeol synthase. Formation of the orysatinyl cation (44) would be expected to occur during initial formation of the C-ring. It is now believed that the 6,6,6 tricyclic precursor to the protosteryl cation (51), proceeds via a 6,6,5 intermediate (50) by a Markovnikov-type addition followed by C-ring expansion (Fig. 4b). ${ }^{54}$ Attack of the alkene at different faces of the bicyclic C8 cation (49) would result in differential production of either the classical protosteryl cation (2) or the orysatinyl cation (44) (Fig. 4b). In the proposed orysatinyl cation (44), both the C8 and C14 methyl groups are in the $\beta$-configuration, explaining the resulting cis $\mathrm{C} / \mathrm{D}$ ring junction of orysatinol (48). This would also imply that the 1,2-shifts of the C8, and C14 methyl groups are not concerted, as both methyl groups must migrate across the same face of the compound. This is not an unreasonable assumption as orysatinol synthase
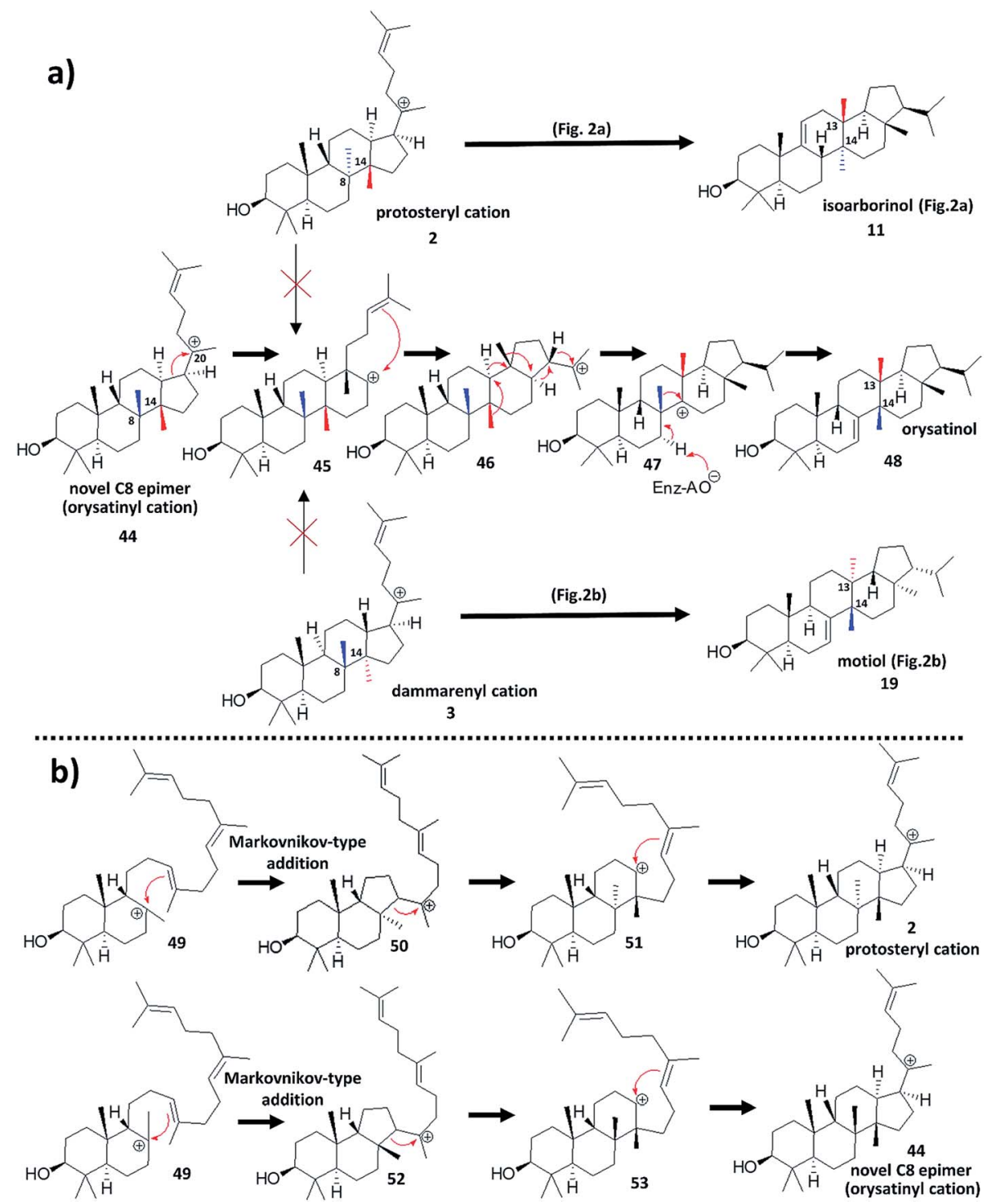

Fig. 4 (a) Orysatinol is not easily rationalizable from either the protosteryl or dammarenyl cations but from a novel cation. (b) The proposed novel tetracyclic cation may differentiate from the protosteryl cation during initial C-ring formation. *Deprotonation steps may be spontaneous in same cases. 


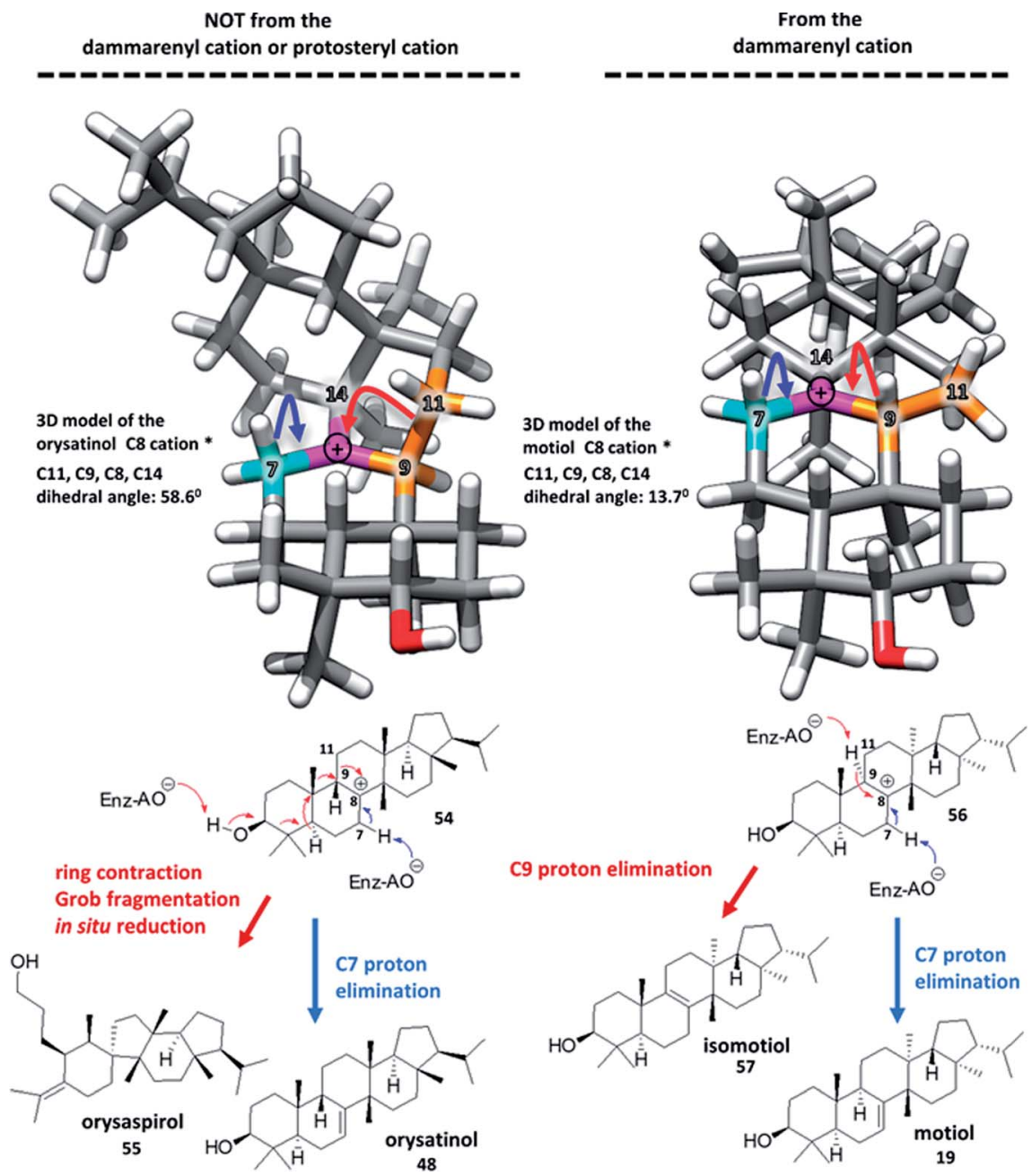

Fig. 5 Structures of orysaspirol, orysatinol, isomotiol, motiol, and the respective parent cations. *Geometry optimised by molecular dynamics, force field: MMFF94, number of steps: 500, algorithm: steepest decent, convergence: $10 \times 10^{-7}$, run by Avogadro version 1.1.1. *Deprotonation steps may be spontaneous in same cases.

(like many OSCs) is a multifunctional enzyme producing several side-products, and this would suggest intermediately cations must be available.

If wide-spread in nature, this novel third cyclisation route would potentially widen the theoretical scope of natural triterpenoid diversity. This is exemplified by the interesting structure of a novel side product of the wild-type orysatinol synthase enzyme, orysaspirol (55). NMR analysis suggests that orysaspirol (55) possesses a highly unusual spirocyclised B/C ring junction, and a seco-A-ring of the type observed in the partially cyclised triterpene marneral..$^{55}$ Generation of orysaspirol (55) can be rationalised from the same pentacyclic C8 parent cation (54) as orysatinol itself, where instead of a C7 elimination to give the alkene, an alternative ring contraction, proceeded by Grob fragmentation gives the spiro-ring junction and seco-A-ring (Fig. 5). It is only plausible to postulate such a mechanism due to the novel stereochemistry of the orysatinol C8 cation (54). This stereochemistry results in the C9-C11 sigma bond being appropriately orientated relative to the empty $\mathrm{p}$ orbital of the C8 carbocation, such that it is reasonable to propose that a ring contraction might be possible (Fig. 5) since the migrating C9-C11 sigma bond takes up the same stereochemical orientation as the $\mathrm{C}-\mathrm{H}$ sigma bond in the wellestablished rearrangement of the dammarenyl cation (3) to form motiol (19). Furthermore, this route might be expected to be entropically favourable because it initiates the fragmentation cascade which opens the A-ring, and possibly thermodynamically favourable because it could relieve steric strain imposed by the cis $\mathrm{C} / \mathrm{D}$ ring junction. In the case of the dammarenyl-derived motiol (19), it is not reasonable to propose an equivalent ring contraction, and this is evident by the existence of isomotiol $(57)^{56}$ which represents an alternative resolution of the motiol parent C8 cation (56) through a C9 hydrogen elimination instead of the $\mathrm{C} 7$ hydrogen elimination observed in the formation of motiol (19) itself (Fig. 5).

\section{Closing remarks}

The intention of this Highlight article is to present the wider contextual significance of orysatinol's (48) structure and 
highlight this potentially important discovery to the wider natural product chemistry community for discussion. Not only does this discovery break a 'textbook dogma', it could widen the potential scope of natural triterpene scaffolds that could exist in nature. Furthermore, it could call into question the reliability of stereochemical assignments of some existing triterpene structures, that are supported by only limited spectroscopic evidence. For example, the reader is asked to imagine a situation where isomotiol (57) had been reported in the literature, but motiol (19) had not. In such a situation, if one was attempting to determine the structure of orysatinol (48) by NMR spectroscopy, and had solved the connectivity of its scaffold, one would likely be strongly biased into believing that they had discovered motiol (19), as this is easily predicted from the parent C8 cation (56) of isomotiol (57).

The isolation of novel triterpene prototypes from nature has been a major contributor in expanding the understanding of the cyclisation process of 2,3-oxidosqualene (1). Orysaspirol (55) is a very minor side product of the wild-type orysatinol synthase enzyme, which was isolated as a result of synthetic biological efforts. It seems unlikely that this product would ever have be isolated though speculative prospecting of the rice plant through traditional natural product extraction workflows. The isolation of the orysaspirol (55), therefore highlights the potential and likely future impact of synthetic biology in the field of natural product chemistry. The stereochemical assignment of orysatinol (48), proven by X-ray crystallography, clearly provides direct experimental evidence to justify more flexibility in the stereochemical interpretation of the biogenic isoprene rule; a rule that has served as a powerful tool to aid structural determination of new triterpene natural products for decades.

\section{Conflicts of interest}

There are no conflicts to declare.

\section{Acknowledgements}

This work was supported by Biological Sciences Research Council (BBSRC)-funded OpenPlant Synthetic Biology Research Centre grant (BB/L014130/1) (M. J. S., A. O.); and the BBSRC Institute Strategic Programme Grant 'Molecules from Nature' (BB/P012523/1) and the John Innes Foundation (A. O.).

\section{Notes and references}

1 R. Xu, G. C. Fazio and S. P. T. Matsuda, Phytochemistry, 2004, 65, 261-291.

2 A. Eschenmoser, L. Ruzicka, O. Jeger and D. Arigoni, Helv. Chim. Acta, 1955, 38, 1890-1904.

3 A. Eschenmoser and D. Arigoni, Helv. Chim. Acta, 2005, 88, 3011-3050.

4 R. Thimmappa, K. Geisler, T. Louveau, P. O'Maille and A. Osbourn, Annu. Rev. Plant Biol., 2014, 65, 225-257.

5 A. E. Osbourn, X. Qi, B. Townsend and B. Qin, New Phytol., 2003, 159, 101-108.
6 T. Moses, K. K. Papadopoulou and A. Osbourn, Crit. Rev. Biochem. Mol. Biol., 2014, 49, 439-462.

7 R. G. Curtis, J. Fridrichsons and A. M. Mathieson, Nature, 1952, 170, 321.

8 W. Voser, M. V. Mijović, H. Heusser, O. Jeger and L. Ruzicka, Helv. Chim. Acta, 1952, 35, 2414-2430.

9 C. S. Barnes, D. H. R. Barton, J. S. Fawcett and B. R. Thomas, J. Chem. Soc., 1953, 576-579, DOI: 10.1039/jr9530000576.

10 D. Arigoni, R. Viterbo, M. Dünnenberger, O. Jeger and L. Ruzicka, Helv. Chim. Acta, 1954, 37, 2306-2322.

11 D. H. R. Barton, Experientia, 1950, 6, 316-320.

12 A. Fürst and Pl. A. Plattner, Helv. Chim. Acta, 1949, 32, 275283.

13 B. A. Hess, Org. Biomol. Chem., 2017, 15, 2133-2145.

14 C. Nanhao, W. Shenglong, S. Lidia, H. B. Andes and W. Ruibo, Angew. Chem., Int. Ed., 2015, 54, 8693-8696.

15 A. B. Banta, J. H. Wei, C. C. C. Gill, J.-L. Giner and P. V. Welander, Proc. Natl. Acad. Sci. U. S. A., 2017, 114, 245-250.

16 V. Domingo, J. F. Arteaga, J. F. Quilez del Moral and A. F. Barrero, Nat. Prod. Rep., 2009, 26, 115-134.

17 P. D. Cárdenas, P. D. Sonawane, J. Pollier, R. Vanden Bossche, V. Dewangan, E. Weithorn, L. Tal, S. Meir, I. Rogachev, S. Malitsky, A. P. Giri, A. Goossens, S. Burdman and A. Aharoni, Nat. Commun., 2016, 7, 10654.

18 W. J. Chin, R. E. Corbett, C. K. Heng and A. L. Wilkins, J. Chem. Soc., Perkin Trans. 1, 1973, 1437-1446, DOI: 10.1039/ p19730001437.

19 H. Lou, X. Li, M. Onda, Y. Konda, M. Urano, Y. Harigaya, H. Takayanagi and H. Ogura, Chem. Pharm. Bull., 1991, 39, 2271-2276.

20 M. L. Oyarzún, J. A. Garbarino, V. Gambaro, J. Guilhem and C. Pascard, Phytochemistry, 1986, 26, 221-223.

21 O. Kennard, L. R. di Sanseverino and J. S. Rollett, Tetrahedron, 1967, 23, 131-148.

22 R. B. Woodward and K. Bloch, J. Am. Chem. Soc., 1953, 75, 2023-2024.

23 A. H. Payne and D. B. Hales, Endocr. Rev., 2004, 25, 947-970. 24 K. U. Wendt, Angew. Chem., Int. Ed., 2005, 44, 3966-3971.

25 W. K. Ulrich, G. E. Schulz, E. J. Corey and D. R. Liu, Angew. Chem., Int. Ed., 2000, 39, 2812-2833.

26 E. J. Corey and S. C. Virgil, J. Am. Chem. Soc., 1991, 113, 40254026.

27 M. Suzuki, T. Xiang, K. Ohyama, H. Seki, K. Saito, T. Muranaka, H. Hayashi, Y. Katsube, T. Kushiro, M. Shibuya and Y. Ebizuka, Plant Cell Physiol., 2006, 47, 565-571.

28 H. R. Bentley, J. A. Henry, D. S. Irvine and F. S. Spring, J. Chem. Soc., 1953, 3673-3678, DOI: 10.1039/jr9530003673.

29 D. H. R. Barton, J. Chem. Soc., 1951, 1444-1451, DOI: 10.1039/jr9510001444.

30 K. Schreiber and G. Osske, Tetrahedron, 1964, 20, 1803-1805. 31 W. D. Nes, R. C. Heupel, M. Benson, A. E. Stafford and W. F. Haddon, J. Chem. Soc., Chem. Commun., 1984, 223225, DOI: $10.1039 / \mathrm{c} 39840000223$.

32 D. Arigoni, O. Jeger and L. Ruzicka, Helv. Chim. Acta, 1955, 38, 222-230. 
33 E. Ménard, H. Wyler, A. Hiestand, D. Arigoni, O. Jeger and L. Ruzicka, Helv. Chim. Acta, 1955, 38, 1517-1529.

34 T. R. Ames, T. G. Halsall and E. R. H. Jones, J. Chem. Soc., 1951, 450-457, DOI: 10.1039/jr9510000450.

35 B. Bischof, O. Jeger and L. Ruzicka, Helv. Chim. Acta, 1949, 32, 1911-1921.

36 D. H. R. Barton, J. Chem. Soc., 1953, 1027-1040, DOI: 10.1039/jr9530001027.

37 T. Kariyone, Y. Hashimoto and S. Tobinaga, Pharm. Bull., 1957, 5, 369-370.

38 S. Nakamura, T. Yamada, H. Wada, Y. Inoue, T. Goto and Y. Hirata, Tetrahedron Lett., 1965, 6, 2017-2022.

39 O. C. Musgrave, J. Stark and F. S. Spring, J. Chem. Soc., 1952, 4393-4397, DOI: 10.1039/jr9520004393.

40 S. David, Bull. Soc. Chim. Fr., 1949, 155-160.

41 S. Koul, T. K. Razdan, C. S. Andotra, A. K. Kalla, S. Koul, S. C. Taneja and K. L. Dhar, Phytochemistry, 2000, 53, 305309.

42 J. M. Beaton, F. S. Spring, R. Stevenson and J. L. Stewart, J. Chem. Soc., 1955, 2131-2137, DOI: 10.1039/jr9550002131.

43 P. Sengupta and H. N. Khastgir, Tetrahedron, 1963, 19, 123132.

44 T. Itoh, T. Shigemoto, N. Shimizu, T. Tamura and T. Matsumoto, Phytochemistry, 1982, 21, 2414-2415.

45 A. Chatterjee, A. B. Kundu, T. Chakrabortty and S. Chandrasekharan, Chem. Commun., 1968, 418-419, DOI: 10.1039/c19680000418.
46 P. Sengupta, S. Ghosh and L. J. Durham, Tetrahedron, 1966, 22, 3469-3476.

47 A. C. Paton, F. S. Spring and R. Stevenson, J. Chem. Soc., 1958, 2640-2645, DOI: 10.1039/jr9580002640.

48 S. Matsunaga, R. Tanaka and M. Akagi, Phytochemistry, 1988, 27, 535-537.

49 J.-P. Declercq, L. Van Puyvelde, N. De Kimpe, M. Nagy, G. Verhegge and R. De Vierman, Acta Crystallogr., Sect. C: Cryst. Struct. Commun., 1991, 47, 209-211.

50 E. J. Corey and J. J. Ursprung, J. Am. Chem. Soc., 1956, 78, 5041-5051.

51 C.-W. Chang, T.-S. Wu, Y.-S. Hsieh, S.-C. Kuo and P.-D. L. Chao, J. Nat. Prod., 1999, 62, 327-328.

52 Z. Xue, Z. Tan, A. Huang, Y. Zhou, J. Sun, X. Wang, R. B. Thimmappa, M. J. Stephenson, A. Osbourn and X. Qi, New Phytol., 2018, 218, 1076-1088.

53 R. Ito, K. Mori, I. Hashimoto, C. Nakano, T. Sato and T. Hoshino, Org. Lett., 2011, 13, 2678-2681.

54 E. J. Corey, S. C. Virgil, H. Cheng, C. H. Baker, S. P. T. Matsuda, V. Singh and S. Sarshar, J. Am. Chem. Soc., 1995, 117, 11819-11820.

55 Q. Xiong, W. K. Wilson and S. P. T. Matsuda, Angew. Chem., Int. Ed., 2006, 45, 1285-1288.

56 H. Singh, V. K. Kapoor, F. Piozzi, S. Passannanti and M. Paternostro, Phytochemistry, 1978, 17, 154-155. 